\title{
Cerebrospinal fluid dynamics in pediatric pseudotumor cerebri syndrome
}

\author{
Afroditi-Despina Lalou ${ }^{1,2}$ (D) - James S. McTaggart ${ }^{3} \cdot$ Zofia H. Czosnyka $^{1} \cdot$ Matthew R Garnett $^{2}$ - Deepa Krishnakumar ${ }^{3}$. \\ Marek Czosnyka ${ }^{1}$
}

Received: 22 May 2019 / Accepted: 17 June 2019 / Published online: 19 July 2019

(C) The Author(s) 2019

\begin{abstract}
Purpose There is a growing body of evidence highlighting the importance of comprehensive intracranial pressure (ICP) values in pseudotumor cerebri syndrome (PTCS). Due to the highly dynamic nature of ICP, several methods of ICP monitoring have been established, including the CSF infusion study. We have performed a retrospective review of the CSF dynamics measurements for all pediatric patients investigated for PTCS in our center and examined their diagnostic value compared with clinical classification.

Methods We retrospectively recruited 31 patients under 16 years of age investigated for PTCS by CSF infusion test. We used the clinically provided Friedman classification 13/31 patients with definite PTCS (group A), 13/31 with probable PTCS (group B), and 5/31 not PTCS (group C), to compare CSF dynamics in the 3 groups.

Results CSF pressure (CSFp) was significantly increased in group A $(29.18 \pm 7.72 \mathrm{mmHg})$ compared with B $(15.31 \pm$ $3.47 \mathrm{mmHg} ; p=1.644 \mathrm{e}-05)$ and $\mathrm{C}(17.51 \pm 5.87 ; p=0.01368)$. The amplitude (AMP) was higher in the definite $(2.18 \pm$ $2.06 \mathrm{mmHg})$ than in group B $(0.68 \pm 0.37 ; p=0.01382)$. There was no in either CSFp or AMP between groups B and C. No lower breakpoint of the AMP-P line was observed in group A but was present in 2/13 and 2/5 patients in groups B and C. In group A, sagittal sinus pressure (SSp) and elasticity were the only parameters above threshold $(p=4.2 \mathrm{e}-06$ and $p=0.001953$, respectively), In group B, only the elasticity was significantly higher than the threshold ( $p=004257)$. Group C did not have any of the parameters raised. The AUC of CSFp, elasticity, and SSp for the 3 groups was $93.8 \%$ (84.8-100\% CI).

Conclusions Monitoring of CSFp and its dynamics, besides providing a more precise methodology for measuring CSFp, could yield information on the dynamic parameters of CSFp that cannot be derived from CSFp as a number, accurately differentiating between the clinically and radiologically derived entities of PTCS.
\end{abstract}

Keywords Idiopathic intracranial hypertension $\cdot$ Pseudotumor cerebri $\cdot$ Intracranial pressure $\cdot$ CSF dynamics $\cdot$ CSF infusion studies

\section{Introduction}

The pseudotumor cerebri syndrome (PTCS) has been principally described as a disease of post-pubertal females with

Afroditi-Despina Lalou

ad143@cam.ac.uk

1 Department of Clinical Neurosciences, Division of Neurosurgery, University of Cambridge, Hills Road, Cambridge CB20QQ, UK

2 Department of Clinical Neurosciences, Division of Neurosurgery, Cambridge University Hospital, Cambridge, UK

3 Department of Pediatric Neurology, Cambridge University Hospital, Cambridge, UK raised BMI and its accurate diagnosis is particularly challenging in the pediatric population. The current intracranial pressure (ICP) thresholds in the pediatric spectrum of the disease (28 cm CSF for obese and/or sedated children, $25 \mathrm{~cm}$ CSF for normal weight and non-sedated children for definite PTCS) [1-3] have been established epidemiologically, being set at the 90th percentile level of ICP recordings obtained in a "normal" pediatric population [4]. There are no adequately powered, randomized studies to reliably confirm that the thresholds are clinically relevant $[1,5-7]$.

There is a growing body of evidence highlighting the importance of long-term ICP monitoring against single "snapshot" manometric measurements. For example, it is wellknown that standard lumbar puncture (LP) measurements can be affected by posture, movement, emotion, anesthetic 
agents, and hypercapnia, which can lead to unreliable estimation of ICP (CSF pressure (CSFp) if it is derived from connecting to the spinal canal rather than the parenchyma) [8-11]. It is particularly important to bear in mind that ICP levels are dynamic [7, 9-11] so a "normal" recorded ICP at a single timepoint may not be a representative value. In adults and children, the assessment of average ICP over more than 20 min ("steady state") is reported to be more reliable than a single opening pressure measurement [5, 10, 12-14] (Fig. 1). Although it is important to estimate ICP more reliably, baseline ICP as a solitary number cannot provide a full assessment for the diagnosis and understanding of the CSF circulatory disorders. This is because it is the production, circulation or reabsorption of CSF that makes ICP dynamic and is likely to be deranged in PTCS. Historically, only around 30\% of these disturbances have been shown to be reflected on steady-state ICP values [7, 15-20]. For these reasons, monitoring of ICP in conjunction with CSF dynamics has long been established in the field [9, 11, 21-23].

At our center, children with suspected PTCS routinely undergo a lumbar infusion study. During the infusion test, they have a LP with measurement of a baseline, longer term CSFp monitoring, and, if the pressure is below approximately $30 \mathrm{mmHg}$, sterile artificial CSF fluid is infused via the spinal needle. The resultant pressure-volume data can be analyzed to give the CSF dynamics variables such as the pulse amplitude of ICP, the compensatory reserve, assessed mainly as the moving correlation coefficient between mean CSF pressure and pulse amplitude (RAP), and the magnitude of slow waves of CSFp [24-26].
Using this technique, children with optic disc edema at our center can be diagnosed accurately with a raised CSFp (definite PTCS) and normal CSFp (probable PTCS more reliably than with standard LP), and we can also characterize the CSF dynamics of each child.

The methodology described has been used in our center and others to report on the profiles of CSF dynamics that are seen in various types of pediatric and adult hydrocephalus [5, 27-30]. By contrast, there is little reported information on the profile of CSF dynamics that characterizes PTCS. In adults with PTCS, it has been reported that there is a dynamic coupling between CSFp and sagittal sinus pressure (SSp), which was demonstrated by performing infusion studies simultaneously with direct cerebral venous pressure monitoring via a catheter [31]. Our previous paper [32], which reported on children referred to our center from 2006 to 2009, remains as the only report on CSF dynamics in pediatric PTCS.

We aimed to investigate the profile of CSF dynamics in pediatric PTCS and to see if it could provide additional, clinically useful insights. For this purpose, we performed a retrospective review of the CSF dynamics for all patients referred to our tertiary pediatric services $(\leq 16$ years old) for consideration of a diagnosis of PTCS from 2006 to 2016 . We have reanalyzed their original CSF infusion study data in an up-todate mathematical model and investigated the CSF dynamics disturbances in definite and probable pediatric PTCS. We have also tested their diagnostic yield compared with the traditional investigations that these children undergo.

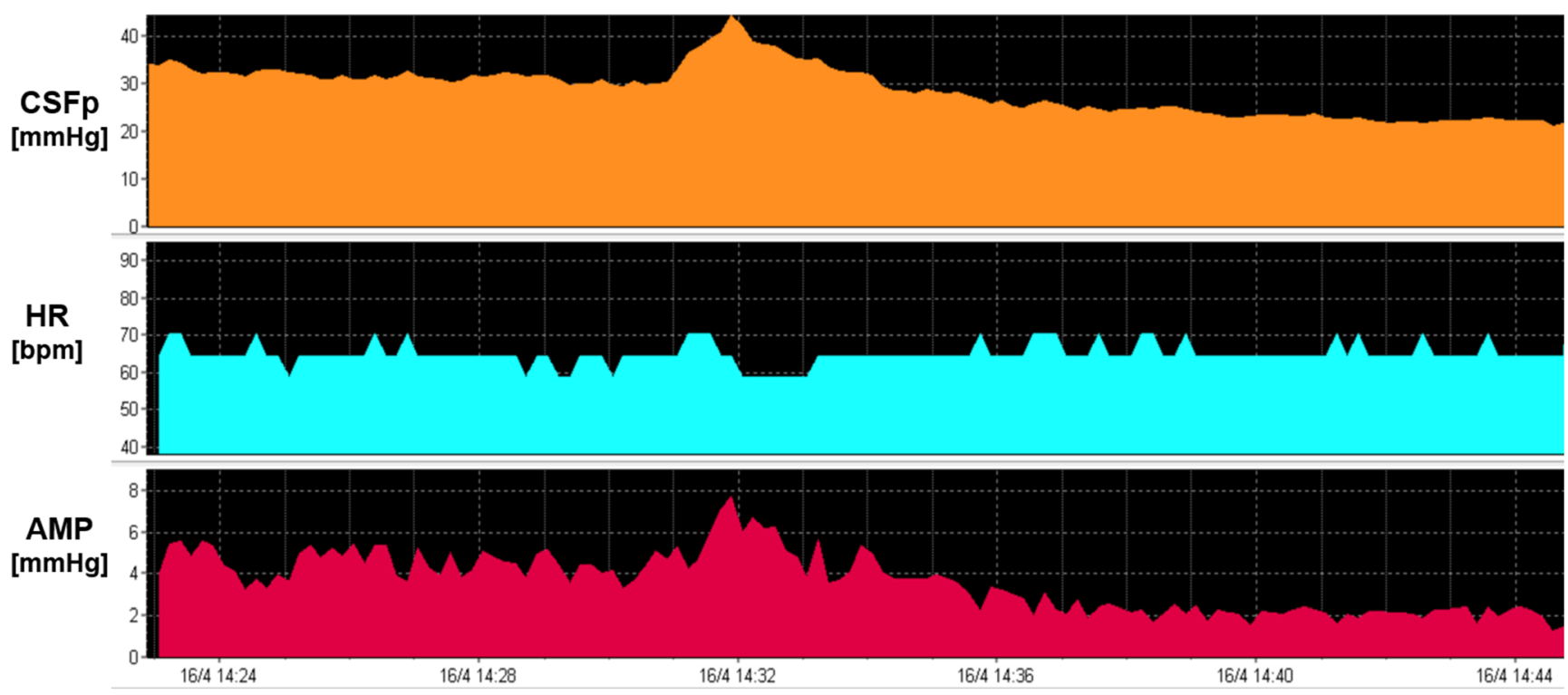

Fig. 1 Variability of CSF pressure. Upper panel: pediatric patient after a LP and connection to the computer for monitoring, showing initially raised CSFp of $32 \mathrm{mmHg}$, spontaneously receding to $20 \mathrm{mmHg}$ after

20 min of monitoring. CSFp cerebrospinal fluid pressure, AMP fundamental amplitude of CSFp, HR heart rate 


\section{Methods}

\section{Patient selection}

Seventy-two children were referred to the pediatric neurology team at Cambridge University Hospital for consideration of a diagnosis of PTCS from 2006 to 2016. Thirty-two children from the cohort were excluded from this paper as they did not undergo an infusion test and/or received a diagnosis other than PTCS. Nine children were excluded (some of whom underwent infusion) because they had PTCS due to a host of medical conditions or medications that had previously been reported to be associated with raised ICP ("secondary PTCS"). In this study, we report the results of the remaining 31 children from the cohort, all of whom underwent CSFp monitoring with or without infusion. The results of 7 of these patients have previously been reported [32].

\section{Patient classification}

All the children who underwent CSF infusion study had papilledema confirmed or excluded by an expert ophthalmologist and their neuroimaging reported by an expert neuroradiologist. We have used here the results of that detailed analysis using the Friedman classification (see Table 1).

Thirteen out of 31 patients were classified as definite PTCS (group A), 14/31 as probable PTCS (group B), and /31 as not PTCS (group C) [1]. In groups A and B, all children had papilledema (one of the requirements) and fulfilled criteria 1-5 and 1-4 in the Friedman classification (see Table 1), whereas none of the children in group $\mathrm{C}$ had papilledema.

We compared the CSF dynamics in these 3 different groups. Because some patients did not have a fluid infusion due to safety reasons (when baseline CSFp was too high; above $30-40 \mathrm{mmHg}$ ), we studied both the baseline and the infusion-derived parameters separately.

\section{Infusion test}

Access to the intrathecal space was gained via LP using a 18gauge Quincke needle, to minimize the effect of the resistance of the needle on measured CSFp. Connection of a fluid-filled pressure transducer (Edwards Lifesciences ${ }^{\mathrm{TM}}$ ) and pressure amplifier (Spiegelberg or Philips) allowed for datapoint recording at a frequency of $30-100 \mathrm{~Hz}$, with the following processing by ICM+ (University of Cambridge Enterprise Ltd). Baseline CSF pressure was monitored for 7-15 min, followed by infusion of Hartmann's solution until the ICP plateaued for 10-15 min or CSFp increased to $40 \mathrm{mmHg}$ or above (at which point infusion was stopped for safety reasons). Details of the computerized CSF infusion test have been reported in various publications [10, 33-35].
Our local procedure involves attempting the infusion test in awake children who can cooperate, with local anesthetic (lidocaine and prilocaine cream) applied at the puncture site $45 \mathrm{~min}$ to $1 \mathrm{~h}$ before the LP. Most children inhale a $50 \%$ nitrous oxide $50 \%$ oxygen mixture during the LP only. If a child is not able to tolerate the above, the procedure is carried on under GA under stable conditions (MAP, ETCO2, and temperature) and with the use of mainly propofol and remifentanil, with a muscle paralyzer, predominantly rocuronium. We analyzed the results in conscious vs GA children to identify potential differences.

All raw infusion test data was re-analyzed in a single unified model for this paper.

In all children who had a standard LP (at their local center) followed by an infusion study (at our center), we sought the relationship between the two values if the tests were carried out within 3 months of each other.

ICM+ software (Cambridge enterprise) [35] was used to calculate the CSF dynamics and compensatory reserve parameters: RAP which is derived from the moving correlation coefficient between ICP and the fundamental amplitude of ICP (AMP) and values $>0.6$ represent depleted compensatory reserve [36-38] and elasticity with values $>0.181 / \mathrm{ml}$ representing depleted compensatory reserve [39, 40]. Different thresholds were also explored for these patients. The sagittal sinus pressure ( $\mathrm{SSp}$ ) is calculated from the theoretical model and does not necessarily reflect the individual's real SSp. Thresholds for SSp and AMP have been reported as $7 \mathrm{mmHg}$ and $2 \mathrm{mmHg}$, respectively [2-7]. All calculated parameters derive from known mathematical models [10, 35, 41, 42], integrated into the software. For PTCS, infusion test cannot reliably estimate resistance to CSF outflow, as sagittal sinus pressure usually rises with CSF pressure, making the value of this parameter overestimated [6].

The lower breakpoint (LBP) and upper breakpoint (UBP) of the amplitude-pressure (AMP-P) regression line was identified, when in existence, in the pediatric PTCS children and the control groups. LBP has mainly been described in NPH, as the breakpoint above which the AMP-P relationship starts becoming linear. The resulting slope of the AMP-P line correlates with elasticity and is a marker of depleted compensatory reserve, if it is $>0.16[43,44]$. The UBP represents a second breakpoint, above which the same linear regression becomes negative and possibly represents a critical point of CSFp, above which cerebral perfusion pressure reaches the lower limit of autoregulation; therefore, ischemia starts to develop, and the pulsations transmitted from the vascular bed decrease.

\section{Ethical approval}

Health Research Authority approval was sought and granted and, in line with this approval and the protocol in the 

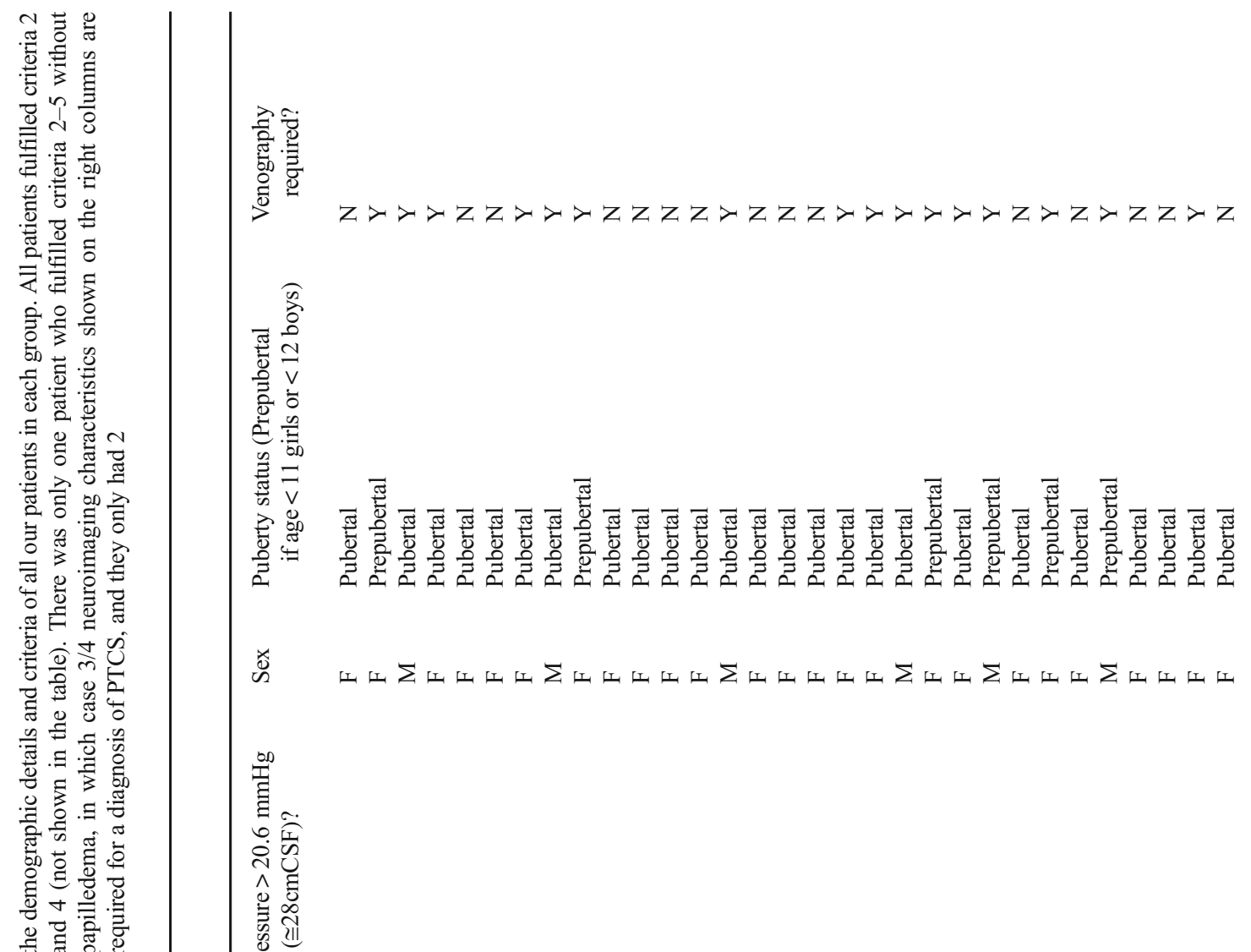

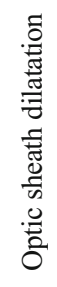

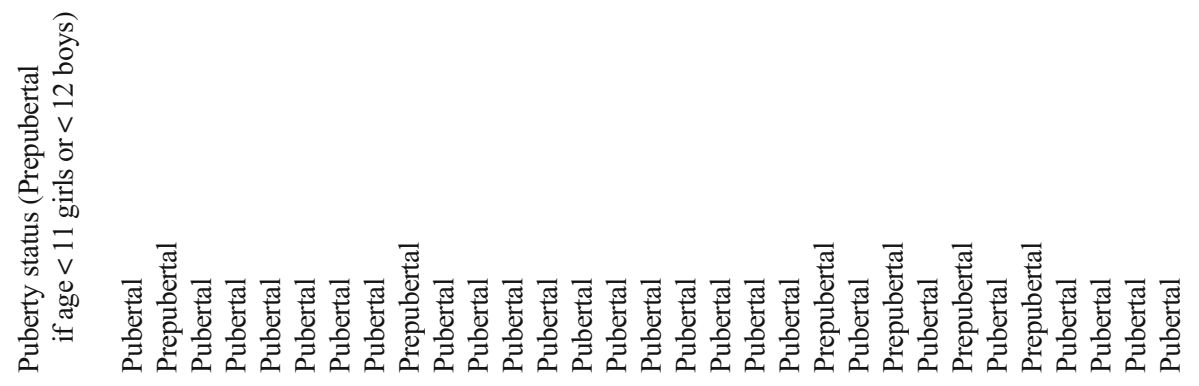

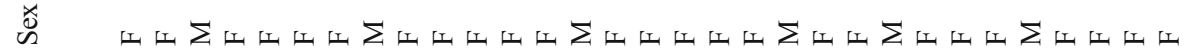

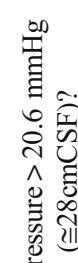

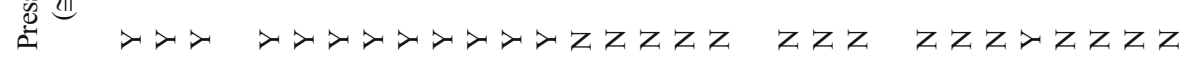

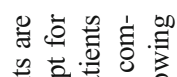

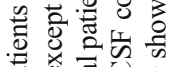

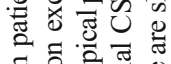

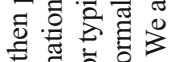

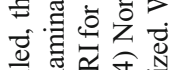

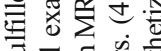

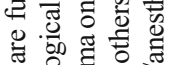

펼 응

.

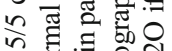

$\Psi$ 乙 苍元范

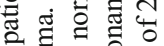

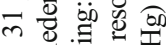

言言总. 总

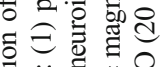

苞苍芴蒫

包䍃

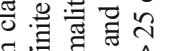

卷责

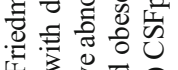

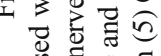

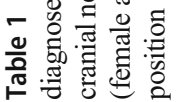

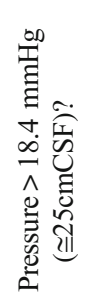
(1) . 


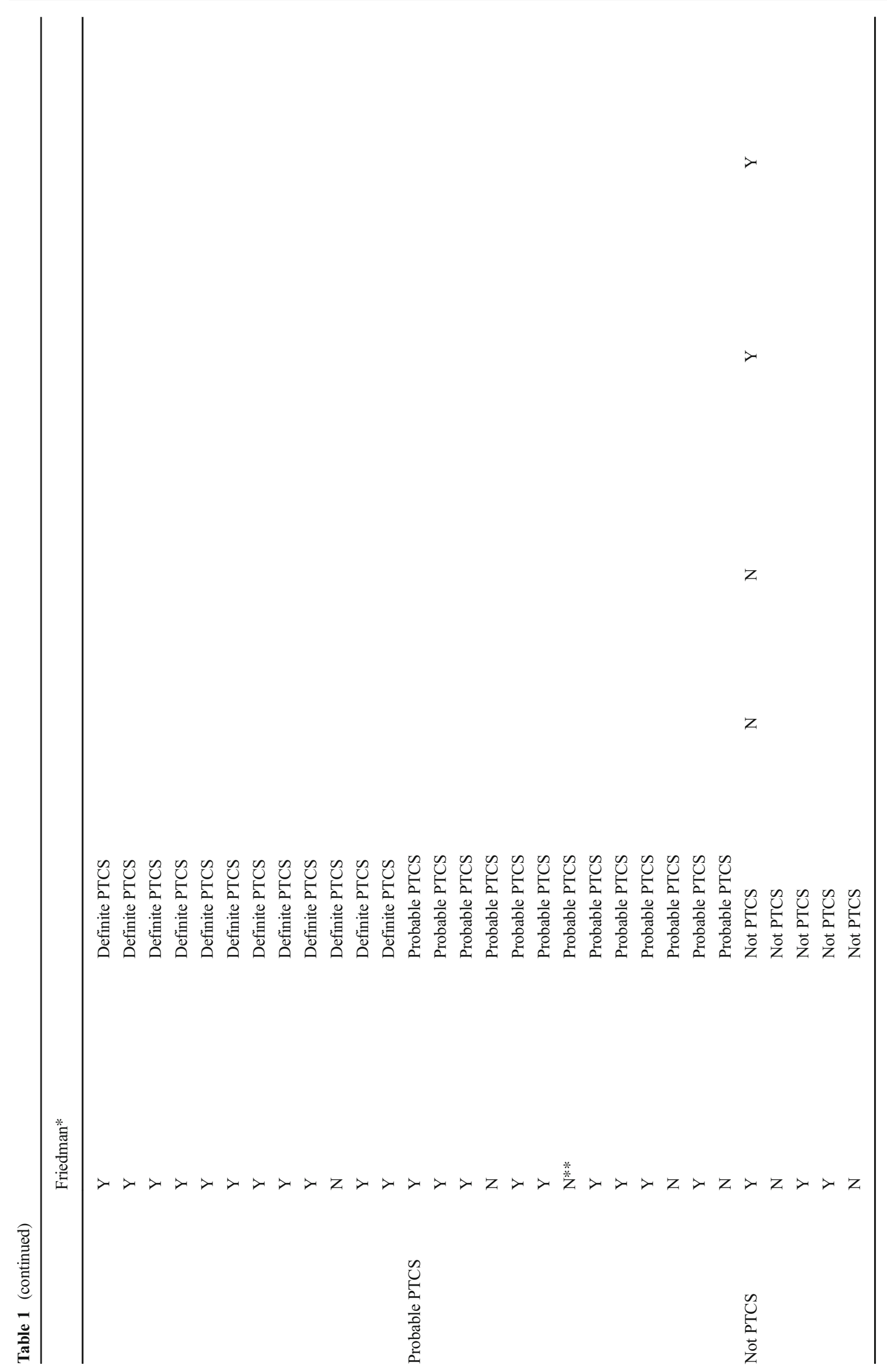


Cambridge University Hospitals NHS Trust, this retrospective study was conducted without separate approval from an ethics committee. All patients were investigated with infusion test within the pediatric neurology clinic and neurosciences department, as a part of routine clinical assessment. They (children/parents) all consented for these studies.

\section{Statistical analysis}

After testing for a normal distribution, non-parametric Wilcoxon's test was used to compare differences in CSF dynamics parameters between the pediatric PTCS groups. The single-sample Wilcoxon test and $t$ test were used to compare CSF dynamics to their reported normative values from the literature. The correlations between different CSF dynamics parameters were sought using Pearson's or Spearman's correlation coefficient, where appropriate. Multiclass ROC was performed to test the diagnostic value of CSF dynamics vs the 3 clinical groups using the package pROC [45]. Plots were created, and data analysis was performed with $\mathrm{R}$ version 3.5.2.

\section{Results}

\section{Demographics}

Detailed demographics, of interest beyond our current focus, such as ethnicity and BMI, are not analyzed in the current paper. All groups were composed of a majority of female (f:m approximately 5:1 in all 3 groups), adolescent, White, pubertal (mean age $12 \pm 3$ years, similar in all 3 groups), obese individuals. There was no obvious difference in the proportion of severely obese patients between the probable and definite PTCS groups. There were more prepubertal, fewer severely obese, and more normal weight patients in the group not diagnosed with PTCS.

\section{Infusion test CSFp and LPs}

Three out of 13 definite PTCS children only had baseline CSFp measurement and drainage, due to significantly raised CSFp (around 40 or more $\mathrm{mmHg}$ ). One patient, who did not have papilledema or sufficient neuroimaging features to make a diagnosis of possible PTCS, was diagnosed with chronic migraine with raised CSFp. These children did not undergo the infusion portion of the test for safety reasons, and as a result, only baseline CSFp and AMP (AMPb), together with RAP variables, could be calculated.

For the 10 children that had a CSFp measured via standard LP prior to the infusion, the correlation between the LP CSFp and the baseline CSFp measured was weak and nonsignificant ( $R=0.30 ; p=0.3977$, Fig. 2). All LPs had been done 2-12 weeks prior to the infusion test.

\section{CSF dynamics in definite, probable, and excluded PTCS diagnosis}

The baseline features of CSF dynamics in the 3 groups are reported analytically in Table 2 .

Figure 3 shows a representative example of CSF infusion test results in Definite PTCS vs probable and not PTCS.

CSFp was by definition increased in definite PTCS (group A) $(29.18 \pm 7.72 \mathrm{mmHg})$, and significantly higher than the probable (group B) $(15.31 \pm 3.47 \mathrm{mmHg} ; p=1.644 \mathrm{e}-05)$ and not PTCS (group C) $(17.51 \pm 5.87 ; p=0.01368)$. The AMP was also higher in the definite $(2.18 \pm 2.06 \mathrm{mmHg})$ than in group $\mathrm{B}(0.68 \pm 0.37 ; p=0.01382)$. However, there was no difference in either CSFp or AMP at baseline between groups $\mathrm{B}$ and $\mathrm{C}(p=0.7028$ and $p=7673$, respectively).

In 2 of the children with definite PTCS, the upper breakpoint of the amplitude-pressure correlation was observed at baseline (UBP at $36.36 \pm 5.01 \mathrm{mmHg}$ ), a phenomenon not observed in the other 2 groups. The observed pattern of CSF dynamics in these 2 cases is demonstrated for one of the patients in Fig. 4. Slow waves, as well as the AMP at plateau and amp-p slope, were similar in all groups. No LBP was observed in group $A$, but it was present in $2 / 13$ and $2 / 5$ patients in groups B and C accordingly. An UBP was present in all groups, with no difference in its value.

There was a significantly higher SSp in definite PTCS vs the other groups $(p=0.0014$ and $p=0.007992)$. In pairwise comparisons of elasticity between the groups, the only significant difference was that elasticity was significantly higher in group A than in group C.

All parameters except for SSp did not follow a normal distribution. In group A, SSp and elasticity were the only ones above their reported threshold (thresholds $7 \mathrm{mmHg}$; $p=4.2 \mathrm{e}-06$ and $0.18 \mathrm{1} / \mathrm{ml} ; p=0.001953$, respectively [2-7]). AMPb was not higher than $2 \mathrm{mmHg}(p=0.6848)$. In group $\mathrm{B}$, only the elasticity was significantly higher than the threshold ( $p=004257)$. For group $C$, all values were not significantly different from the published normal range. These main CSF dynamic parameters of the 3 groups are illustrated in Fig. 5.

\section{Relationship between the CSF dynamics parameters in groups $A-C$}

SSp tended to show a correlation with baseline CSFp in groups $\mathrm{A}(R=0.60 ; p$ value $=0.05034)$ and $\mathrm{B}(R=0.59 ; p$ value $=0.03403)$, but not in group $\mathrm{C}(R=-0.016$; $p$ val$\mathrm{ue}=0.9833)$. Similarly, elasticity showed a significant correlation with $\mathrm{SSp}$ in group $\mathrm{A}(R=0.67 ; p$ value $=0.02807)$ and group $\mathrm{B}(R=0.59 ; p$ value $=0.03193)$, and not in group $\mathrm{C}$ (negative correlation, not significant). There was no correlation between CSFp and RAP or AMP with SSP and elasticity. 
Fig. 2 Discordance between CSFp derived from LP performed either right before the CSF infusion study or within 3 months before the infusion study

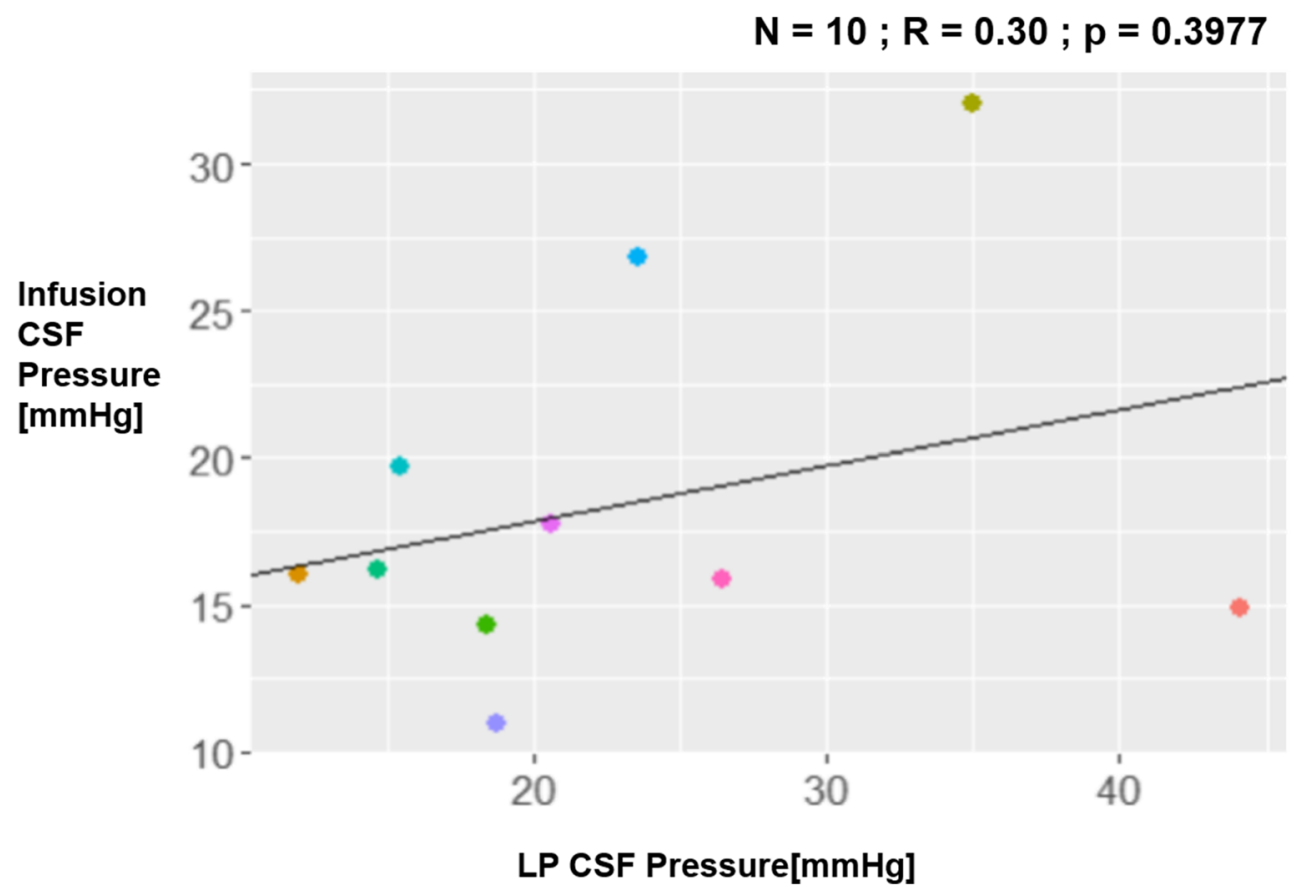

\section{ROC analysis between clinical classification and CSF dynamics in pediatric PTCS}

No baseline or infusion-derived parameter on its own could give a satisfactory separation between the 3 groups. However, given the significant differences in CSFpb, SSp, and elasticity, and secondarily in AMPb and CSFpp, the best AUC was when the correlation between CSFpb, SSp, and elasticity was integrated into a linear model, with a resulting AUC 93.8\%, with $84.8-100 \%$ CI $(95 \%$ CI) in multiclass ROC analysis among the 3 different groups (Fig. 6).
Table 2 Baseline and infusion-based CSF dynamics parameters of the 3 clinically classified pediatric PTCS groups. Values are represented as mean \pm SD. A different number of patients $(N)$ is shown in each row for group A, since only baseline parameters were monitored in 3/14 patients. RAP, index of compensatory reserve, from the correlation of
AMP and ICP. CSFpb, CSF pressure at baseline. AMPb, fundamental amplitude of ICP at baseline. SSp, sagittal sinus pressure. CSFpp, CSFp at plateau. AMPp, AMP at plateau. AMP-P slope, slope of the amplitudepressure line. LBP, lower breakpoint of the amplitude-pressure line. UBP, upper breakpoint of the amplitude-pressure line

\begin{tabular}{|c|c|c|c|c|c|c|}
\hline Variable & A: definite PTCS & $\begin{array}{l}\text { B: probable } \\
\text { PTCS }(N=13)\end{array}$ & $p$ value $\mathrm{A}$ and $\mathrm{B}$ & C: $\operatorname{not}$ PTCS $(N=5)$ & $p$ value $\mathrm{A}-\mathrm{C}$ & $p$ value $\mathrm{B}$ and $\mathrm{C}$ \\
\hline Baseline CSFp (mmHg) & $\begin{array}{c}29.18 \pm 7.72 \\
\quad(N=13)\end{array}$ & $15.31 \pm 3.47$ & $1.644 e-05$ & $17.51 \pm 5.87$ & 0.01368 & 0.7028 \\
\hline $\mathrm{AMPb}(\mathrm{mmHg})$ & $2.18 \pm 2.06(N=13)$ & $0.68 \pm 0.37$ & 0.01382 & $0.89 \pm 1.03$ & 0.1433 & 0.7673 \\
\hline Baseline RAP & $0.58 \pm 0.3(N=13)$ & $0.46 \pm 0.18$ & 0.2813 & $0.37 \pm 0.12$ & 0.2075 & 0.4016 \\
\hline Elasticity $(1 / \mathrm{ml})$ & $0.36 \pm 0.19(N=10)$ & $0.39 \pm 0.26$ & 0.9505 & $0.15 \pm 0.06$ & 0.002671 & 0.1031 \\
\hline $\mathrm{SSp}(\mathrm{mmHg})$ & $\begin{array}{c}18.99 \pm 4.08 \\
(N=10)\end{array}$ & $9.55 \pm 11.9$ & 0.0014 & $8.65 \pm 1.17$ & 0.007992 & 0.6928 \\
\hline CSFpp (mmHg) & $\begin{array}{c}32.89 \pm 2.92 \\
(N=10)\end{array}$ & $25.42 \pm 4.47$ & 0.0002243 & $25.25 \pm 6.1$ & 0.03996 & 0.775 \\
\hline AMPp (mmHg) & $2.1 \pm 1.11(N=10)$ & $1.55 \pm 0.86$ & 0.2264 & $2.13 \pm 1.86$ & 0.953 & 0.8436 \\
\hline CSFpp-CSFpb (mmHg) & $7.44 \pm 2.73$ & $10.11 \pm 4.06$ & 0.1661 & $7.74 \pm 2.9$ & 0.953 & 0.3873 \\
\hline $\mathrm{AMPp}-\mathrm{AMPb}(\mathrm{mmHg})$ & $0.93 \pm 0.7$ & $0.88 \pm 0.7$ & 0.99 & $1.24 \pm 1.15$ & 0.6787 & 0.775 \\
\hline Amp-p slope & $0.15 \pm 0.09(N=10)$ & $0.09 \pm 0.05$ & 0.1621 & $0.13 \pm 0.08$ & 0.8539 & 0.3233 \\
\hline LBP $(\mathrm{mmHg})$ & $\mathrm{NA}(N=0)$ & $10.5 \pm 2.12(N=2)$ & NA & $15.5 \pm 3.53(N=2)$ & NA & 0.3333 \\
\hline UBP $(\mathrm{mmHg})$ & $36.36 \pm 5.01(N=8)$ & $34.5 \pm 11.39(N=4)$ & 0.99 & $29.5 \pm 7.78(N=2)$ & 0.5714 & 0.8 \\
\hline Slow waves at baseline $(\mathrm{mmHg})$ & $1.16 \pm 1.43(N=13)$ & $0.83 \pm 0.85$ & 0.8798 & $0.79 \pm 0.56$ & 0.99 & 0.99 \\
\hline Slow waves at plateau $(\mathrm{mmHg})$ & $3.21 \pm 2.51(N=10)$ & $4.9 \pm 7.07$ & 0.7501 & $1.5 \pm 1.33$ & 0.2828 & 0.5427 \\
\hline
\end{tabular}




\section{Start of infusion}

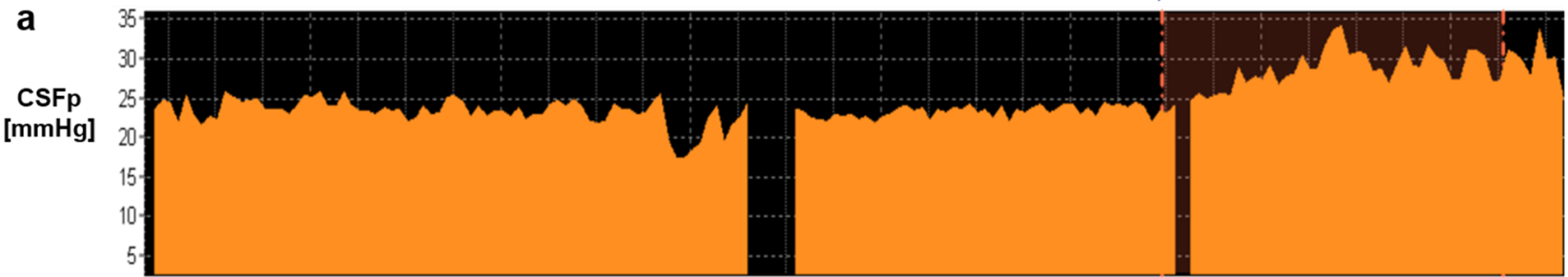

AMP

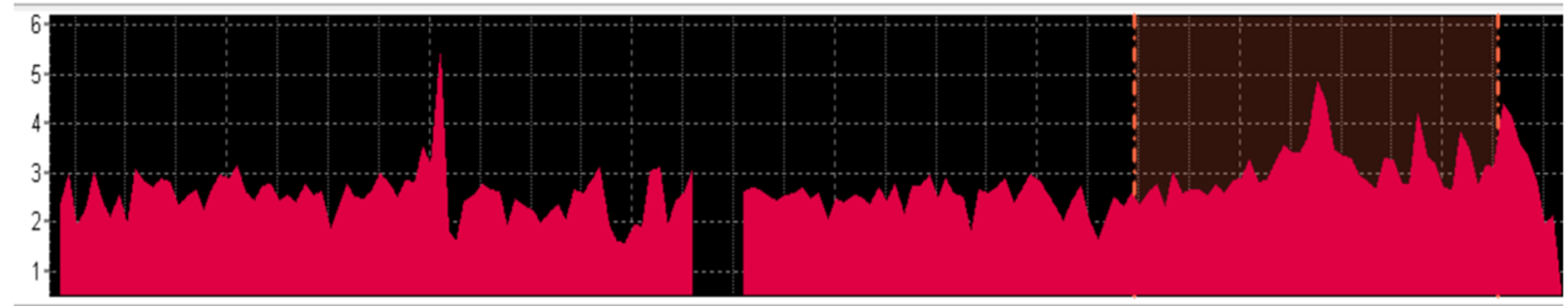

RAP

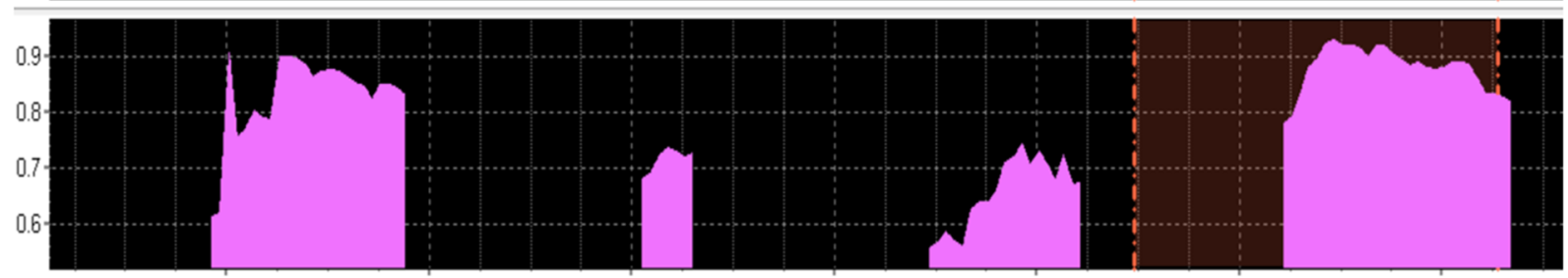

b

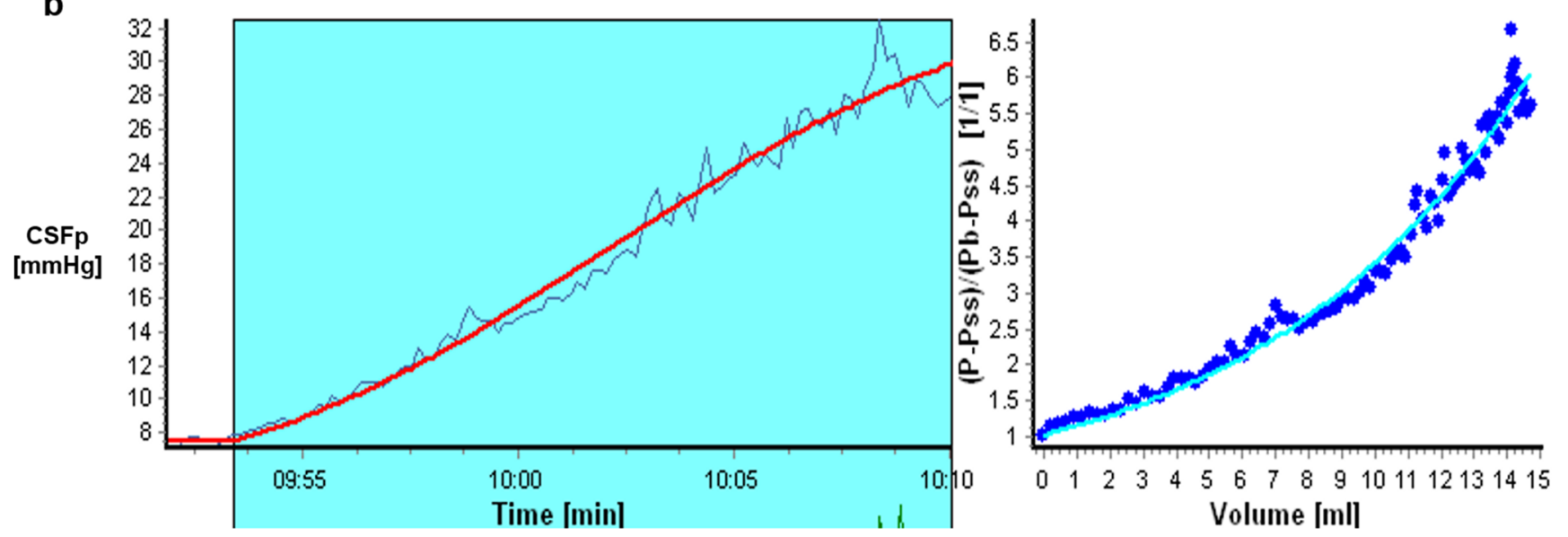

Fig. 3 Representative example of a CSF infusion study and analysis of CSF dynamics on a pediatric patient with definite, active PTCS. a Infusion study recording. A 10-15-min baseline is monitored to ensure stable baseline pressure. The start of infusion is indicated with an arrow and the infusion period is highlighted after the start on the right end. CSFp is elevated, usually $>20 \mathrm{mmHg}$, with low resistance to CSF outflow demonstrated from the generally low plateau of CSFp during infusion. AMP is linearly related to CSFp, with a high correlation coefficient at baseline (RAP >0.4), indicating depleted compensatory reserve. RAP is not always reliable in such short recordings, as shown by the artifacts in AMP, corresponding to gaps in RAP calculation. b Analysis of CSF dynamics in ICM+. Left curve: the solid, curved line represents the theoretical model representing the response of CSFp to infusion at each

time- baseline, during infusion and plateau. The dotted data points represent the calculations performed by the interpreter of the CSF test and which should optimally fit the theoretical mode. Right curve: The solid line represents a similar model, where the calculations of the user should fit the pressure-volume curve as proposed by Marmarou ([46]) and integrated in ICM+. The dotted data points result from the user-performed analysis of the test. CSFp CSF pressure, AMP fundamental amplitude of CSFp. The pressure of the sagittal sinus and elasticity among others is calculated from this mathematical model and are most reliable when the 2 curves coincide. RAP, the correlation between AMP and CSF pressure, when available reliably at baseline, is typically elevated, showing depleted pressure-volume compensation

\section{Influence of GA}

Overall, CSFp monitoring with or without infusion was performed under GA on 6/13 patients in group A, 4/13 in group B and $1 / 5$ in group $\mathrm{C}$.

Within the groups, CSFpb was higher in the definite PTCS group that received GA $(34.8 \pm 7.44$ vs $23.06 \pm 5.43 ; p=$ 0.0452); however, when the 3 children with very high baseline pressures (who underwent drainage and no infusion study) were removed from the analysis, the difference was no longer 


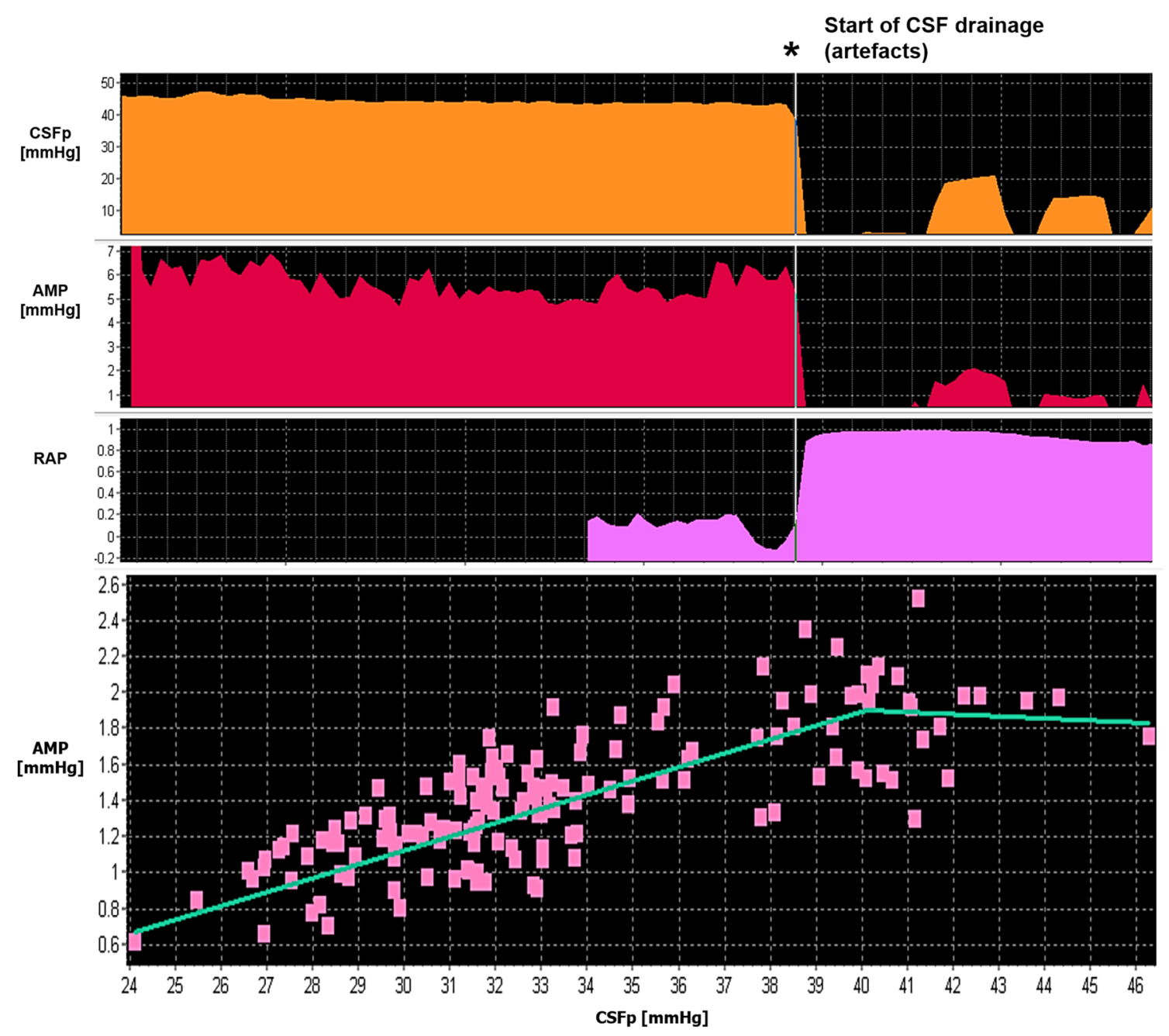

Fig. 4 Upper panel: critically high CSFp ( $42 \mathrm{mmHg}$ ), monitored in operating theaters under general anesthesia. Note that RAP is initially lower, representing possible ischemia cause by low cerebral perfusion pressure (CPP). Mean arterial blood pressure was $65 \mathrm{mmHg}$; therefore, CPP was $23 \mathrm{mmHg}$. During CSF drainage, the positive correlation between AMP and CSFp was restored, and RAP became positive at the higher end, representing depleted compensatory reserve. It can be

present. There were no other differences in the definite PTCS group. In the probable PTCS group, only SSp and elasticity appeared to be lowered by GA $(0.12 \pm 3.48$ vs $13.16 \pm 6.59$; $p=0.006851$ and $0.11 \pm 0.08$ vs $0.53 \pm 0.16 ; p=0.006774$. The amplitude of slow waves was consistently suppressed by GA at baseline and plateau within all groups.

\section{Discussion}

The diagnostic implications of measuring the full set of CSF dynamics parameters in pediatric PTCS are highlighted in this paper, in order to provoke further investigation and to encourage the use of this approach more widely in clinical practice. CSF dynamics could provide information on the contents of observed that CSFp, AMP, and HR signals after the start of drainage contain a lot of artifacts. Lower panel: the transition CSFp point between low CPP and restoration of normal CPP represents the upper breakpoint of the amplitude-pressure regression line, above which the linear correlation between AMP and CSFp is lost and tends to become negative, as shown by the negative RAP in the upper panel

CSFp that are not obvious from CSFp as a solitary number, accurately differentiating between the clinically and radiologically derived entities of PTCS.

We have highlighted that (A) a single recording of a raised CSFp is not necessarily a representative value for a patient's ICP and (B) a raised CSFp value does not give a full understanding of a patient's CSF circulation, including venous drainage. Despite its limitations, we acknowledge that CSFp is a diagnostic criterion in current guidelines and we have therefore described our precise methodology for maximizing the reliability of this single CSF dynamics variable. This methodology includes the measurement of "steady-state" CSFp ([8, 9, 42]) and simultaneous measurement of the blood circulation with CSF pressure recordings, as it is known that there is a complicated interaction between the CSF circulation and the cerebral 
a Mean CSFp and SSp

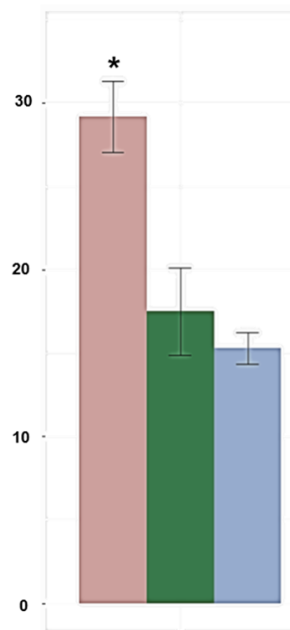

$\mathrm{CSFpb}[\mathrm{mmHg}]$

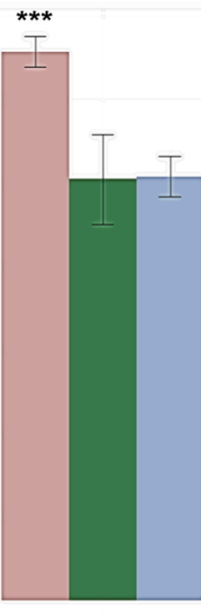

CSFpp [mmHg]

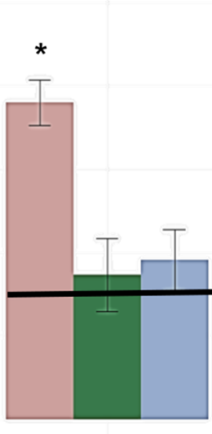

$\mathrm{SSp}[\mathrm{mmHg}]$ b Mean AMP \& compensatory reserve parameters

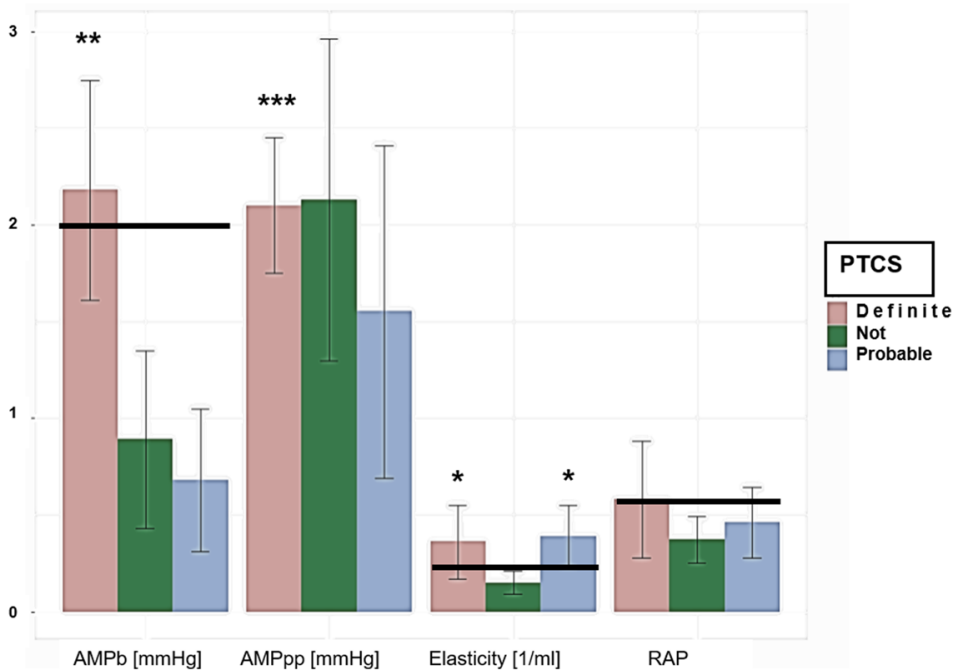

Fig. 5 CSF infusion study results. Summary data of CSF infusion study results according to final diagnosis. Values are represented as mean \pm SEM. Normal thresholds for each parameter are indicated with the horizontal black lines. a CSF pressures: CSFp at baseline and plateau and SSp, as calculated during infusion. b AMP at baseline and plateau, as well as compensatory reserve coefficients RAP and elasticity. The threshold for AMPp, $4 \mathrm{mmHg}$, is not shown since it is much higher than the average in the image and outside the scale for our calculations. The single asterisk indicates significantly higher mean than the other

groups and from the normal threshold, double asterisks indicate significantly higher than the other groups but not from the threshold, and triple asterisks denote the difference in the number of patients in group A ( $N=14$ vs $N=11)$, due to the fact that 3 patients only had baseline values; hence, $\mathrm{AMPb}$ is higher than AMPp in that group. CSFpb CSF pressure at baseline, CSFpp CSF pressure at plateau, SSp sagittal sinus pressure, $\mathrm{AMPb}$ fundamental amplitude of ICP at baseline, AMPp AMP at plateau (mmHg), RAP compensatory reserve index

Studies have already shown that there is a lack of reliability blood circulation in CSF disorders ([7, 9, 43]). We also aim to minimize the impact of other confounding factors such as stress, position, and sedation $[1,4,11,47]$. These factors and how they influence the CSFp cannot be studied and comprehended without analysis of the CSFp components, preferably with cerebral multi-modality monitoring [11, 25, 42]. in the manometry reading of a CSFp $[8,11]$, something which is also partially shown by the lack of correlation between manometry and baseline CSFp from a computerized infusion test in our cohort. However, these readings were not taken at a very close time, but up to 3 months apart. Nonetheless, they
Fig. 6 Area under the curve (AUC) and 95\% CI among Friedman classification of groups A-C and CSF dynamics, in particular the linear model of interaction of CSFp at baseline, elasticity, and sagittal sinus pressure

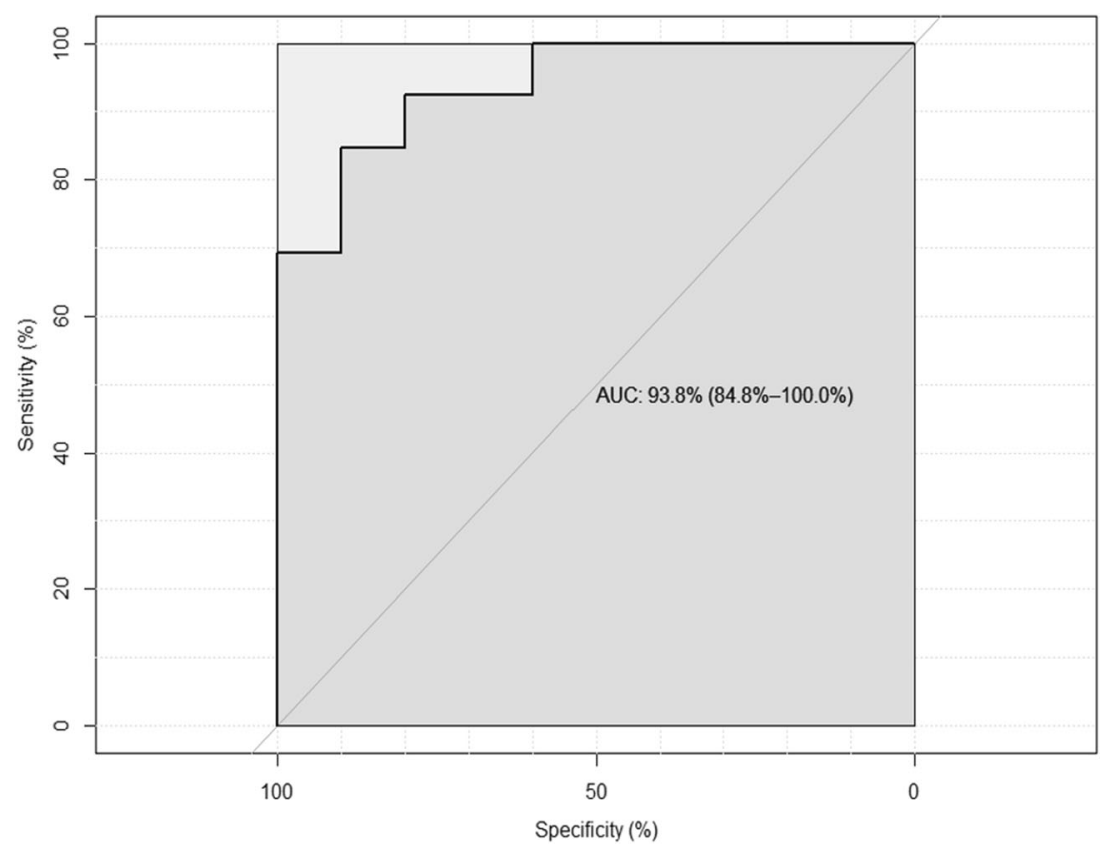


represent the clinical reality of trying to make a diagnosis and a treatment plan from separated LPs and/or drainages, often weeks and month apart from each other.

The CSFp at baseline was by definition raised $>20 \mathrm{mmHg}$ in pediatric PTCS and on average in our group $>25 \mathrm{mmHg}$. In the group of children with definite PTCS, there were 3 children with a CSFp $>30 \mathrm{mmHg}$, and 2 of them had critical levels of CSFp with compromised cerebral perfusion. Although infusion was not possible in these children, the CSF dynamics parameters that could be measured were revealing; a pattern of negative AMP-CSFp relationship was observed, indicating low-cerebral perfusion pressure. It has been reported in traumatic brain injury that when ICP reaches critical levels (usually $>25 \mathrm{mmHg}$ in head injury), the AMP-ICP relationship becomes negative [9, 11], which appears to also be the case with these 2 patients. However, more data are needed in order to confirm this pattern and association in pediatric PTCS. These cases, although seemingly rare, highlight the importance of an extended multiparametric monitoring of the cerebral circulation, including systemic arterial pressure and possibly cerebral autoregulation, blood flow, and oxygenation. It would be interesting, for future purposes, to adopt at a research setting initially a multiparameter monitoring approach, especially in suspicion of serious disease and explore the clinical implications of possible cerebral hypoperfusion.

The difficulty in diagnosing and classifying pediatric PTCS lies in the probable group, where the CSFp was not raised (average $15 \mathrm{mmHg}$ ) and did not differ to the children in whom PTCS was excluded. In these cases, it is of significance to investigate the dynamics of the CSFp. Neither CSFp nor AMP at baseline seemed to help in differentiating between those probable and not PTCS. However, both elasticity and SSp were significantly lower in group C and could potentially provide a key in the differentiation between the two groups. Both elasticity and SSp were elevated in children with definite as well as probable PTCS, showing further potential markers of the disease besides CSFp. However, it is important to bear in mind that both of these parameters are subject to calculation errors and SSp represents a value derived from mathematical modeling of the CSF circulation and not the patient's actual SSp. Unfortunately, it is not possible to understand or confirm what the probable PTCS group represents, as compared with definite PTCS and normal children, until at least some further follow-up and perhaps investigations are performed. Higher SSp as well as higher elasticity could perhaps highlight a tendency or the beginning of the syndrome that has not reached the severity of the definite group.

Besides elasticity and SSp, and partially RAP, none of the other infusion-derived parameters were above the reported normal thresholds ( 0.6 for RAP-lowered to 0.4 for the this cohort of patients, 0.16 for the amp-p line, $4 \mathrm{mmHg}$ for AMPp, $10-13 \mathrm{mmHg} * \mathrm{~min} / \mathrm{ml}$ for Rout), with the exception of slow waves of CSFp at plateau, the magnitude of which exceeded $1.5 \mathrm{mmHg}$ in groups $\mathrm{A}$ and $\mathrm{B}$, but not in group $\mathrm{C}$. Also, the absolute rise in CSFp during infusion was the same in all groups. This rise, divided by infusion rate, denotes the resistance to CSF outflow. As PTCS SSp follows the rise in CSFp, this way of estimation gives an overestimated value of resistance. Nevertheless, in PTCS, CSF circulation is probably normal, as the gradient CSFpp-CSFpb is low [15].

For slow waves in particular, there is no well-described threshold in any of the CSF disorders [24, 26, 48]. Although the differences in the magnitude of slow waves between groups were not significant, there seems to be a tendency of higher magnitude in group $\mathrm{A}$ than in groups $\mathrm{B}$ and $\mathrm{C}$, group $\mathrm{C}$ possibly having the lowest magnitude. Perhaps an increased magnitude of slow waves when the CSF circulation is challenged could reveal a disturbed CSF circulation and assist in differentiating between definite, probable PTCS and mimics. This will need further confirmation. Another finding that could provide interesting information in excluding or confirming PTCS is the presence or absence of a LBP in the AMP-P line. None of the children in the definite group presented with such a breakpoint, whereas it was observed in 2 children for each of the other groups.

There was a correlation between CSFpb and SSp, but not CSFpp. This finding could be similar to what has been shown in adults with PTCS [31], whereby SSp and CSFp are coupled at baseline and during infusion. This relationship, however, cannot be validated in children without direct SSp measurement and there is ethical equipoise currently between the risks and benefits of exposing them to invasive cerebral venous investigation. The correlation between $\mathrm{SSp}$ and elasticity found mainly in our definite PTCS patients could also demonstrate part of the pathophysiology of PTCS, whereby "faulty" venous sinuses allow the transmission of the CSF pressure onto the sinuses, leading to a coupling of the two pressure and thereby a "stiffer" brain. It is therefore possible in children with normal CSFp but abnormal CSF dynamics (e.g., a depleted compensatory reserve) or an abnormal cerebral venous sinus system [5] that CSFp levels can rise with minimal perturbation of the current equilibrium state.

It was intriguing to find out that no single CSF pressure or dynamics parameter on its own could accurately differentiate between the 3 groups with an AUC $>0.80$. Our findings from the ROC analysis show a potential overlap between diagnosis and classification from clinical examination and neuroradiology, with CSF dynamics parameters, in particular, CSFpb, SSP, and elasticity. The main discrepancy between CSF dynamics and the Friedman classification lie in the patient in group $\mathrm{C}$ with no papilledema and disturbed CSF dynamics and in group $\mathrm{B}$, where there is a lot of heterogeneity among the patients. We therefore could speculate that monitoring and infusion, not just baseline parameters, are possibly essential for accurate patient classification and characterization of the disturbance in the CSF circulation. In this context, it is of interest to look into the case of one out of the 5 patients without PTCS, whose CSFp was $27 \mathrm{mmHg}$ and 
did not meet the criteria for PTCS from the Friedman classification. Therefore, in the absence of papilledema, even a confirmed intracranial hypertension perhaps is not synonymous to the actual syndrome and the venous compartment in such cases could merit further investigation. Moreover, it may be possible to distinguish between these two entities both clinico-radiologically, as well as with monitoring of CSF dynamics, and more patients will be needed to make these distinctions.

Finally, the influence of GA on CSF dynamics could not be clearly demonstrated in this small cohort. It has been reported in NPH and TBI patients that GA possibly has no effect on baseline CSFp or elasticity, but significantly dampens the magnitude of slow waves [25]. This study was derived from the same center and therefore included the same GA protocol as the NPH group (propofol + remifentanil and a muscle relaxant, usually rocuronium, naturally with different doses in adults vs pediatric patients). The fact that CSFpb was increased in definite PTCS under GA vs conscious children could be a random finding, or show that anesthetic agents could increase CSFp in pediatric PTCS patients, which is not justified from the literature on the influence of anesthetic agents such as propofol on cerebral blood flow and metabolism. Additionally, this finding, as well as similar previous reports [4, 25, 47], could highlight that children needing GA are usually the ones with a higher BMI, that in our experience is less likely to tolerate a LP, and could reflect the known relationship with BMI in PTCS severity as well as the influence of increased abdominal pressure on CSFp. Preliminarily derived from our set, 7/14 children needed GA in group A, $3 / 3$ with CSFp $>30 \mathrm{mmHg}$. In comparison, our adult and pediatric hydrocephalus children normally require GA when their symptoms are very prominent, which prohibits them from complying with investigations. Similarly, the lower SSp and elasticity in anesthetized probable PTCS patients could mean several things, but this study is not adequately powered to identify if this effect is real. Notably, 14/39 (36\%) of our total children (including the ones diagnosed with secondary PTCS and were excluded from this analysis) that underwent an infusion test had GA during the investigation. This percentage was lower compared with a reported national average of $45 \%$ [2], and we utilized our longer term average CSFp technique that allowed for reliable CSF pressure measurement on a truly continuous scale.

Limitations Our main limitation is the small number of patients in each group and subgroup. Although we were able to show statistically significant results with $p<0.001$, suggesting that the effect sizes are large, analysis of a larger cohort is needed to generalize these results to all children with PTCS. Even though we tried to avoid further confusion by excluding secondary PTCS and selecting well-matched control groups, there were still a lot of different patterns and cases that will need further elaboration in the future, as well as more thoroughly designed studies on the influence of GA on CSF dynamics.

Connected to the above is the fact that to date we have no definitive normative data for CSF dynamics in children. We only performed an infusion test in 5 children without PTCS - initially, they were considered to have a disc appearance that was considered to be borderline for edema, but this was later clarified and confirmed to not be disc edema. As such, the "abnormal" thresholds for the CSF dynamics parameters have mainly been described in hydrocephalus and TBI. From this preliminary study, thresholds appear similar; however, more studies, adequately powered, are needed to validate these parameters.

RAP has got a calculation window of $4 \mathrm{~min}$, and in the short time of the infusion test, the smallest artifacts could make the calculation unreliable; however, calculation without artifacts was possible in all 31 patients. Longer term monitoring is preferred, and RAP is most reliable then; however, a good 10-min baseline with stable variables could give us an estimation of RAP and the compensatory reserve. The magnitude of slow waves is strongly influenced by GA [25] and would require further investigation in awake pediatric PTCS patients.

\section{Conclusion}

CSF dynamics in PTCS show a peculiar profile: baseline CSF pressure is elevated and estimated sagittal sinus pressure is elevated. Elasticity and compensatory reserve are depleted. CSF circulation is probably undisturbed. Very high CSF pressure may contribute to low cerebral perfusion, exposing patients to chronic sub-acute ischemia.

\section{Compliance with ethical standards}

Health Research Authority approval was sought and granted and, in line with this approval and the protocol in the Cambridge University Hospitals NHS Trust, this retrospective study was conducted without separate approval from an ethics committee. All patients were investigated with infusion test within the pediatric neurology clinic and neurosciences department, as a part of routine clinical assessment. They (children/parents) all consented for these studies.

Conflict of interest Author MC has a partial financial interest in licensing ICM+ software (Cambridge Enterprise).

Open Access This article is distributed under the terms of the Creative Commons Attribution 4.0 International License (http:// creativecommons.org/licenses/by/4.0/), which permits unrestricted use, distribution, and reproduction in any medium, provided you give appropriate credit to the original author(s) and the source, provide a link to the Creative Commons license, and indicate if changes were made. 


\section{References}

1. Friedman D, Liu GT, Digre KB (2013) Revised diagnostic criteria for the pseudotumor cerebri syndrome in adults and children. Neurology 81:1159-1165

2. Matthews Y-Y, Dean F, Lim MJ et al (2017) Pseudotumor cerebri syndrome in childhood: incidence, clinical profile and risk factors in a national prospective population-based cohort study. Arch Dis Child 102:715 LP-715721

3. Moavero R, Sforza G, Papetti L, Battan B, Tarantino S, Vigevano F, Valeriani M (2018) Clinical features of pediatric idiopathic intracranial hypertension and applicability of new ICHD-3 criteria. Front Neurol 9:819

4. Avery RA, Shah SS, Licht DJ, Seiden J (2010) Reference range for cerebrospinal fluid opening pressure in children. N Engl J Med 363(9):891-893

5. Kasprowicz M, Lalou DA, Czosnyka M, Garnett M, Czosnyka Z (2016) Intracranial pressure, its components and cerebrospinal fluid pressure-volume compensation. Acta Neurol Scand 134:168-180

6. Pickard JD, Czosnyka Z, Czosnyka M et al (2008) Coupling of sagittal sinus pressure and cerebrospinal fluid pressure in idiopathic intracranial hypertension-a preliminary report. Acta Neurochir Suppl 102:283-285

7. Pickard J (1988) Physiology and pathophysiology of the cerebrospinal fluid. J Neurol Neurosurg Psychiatry 51:469-470

8. Cartwright C, Igbaseimokumo U (2015) Lumbar puncture opening pressure is not a reliable measure of intracranial pressure in children. J Child Neurol 30(2):170-173

9. Czosnyka M, Hutchinson PJ, Balestreri M et al (2006) Monitoring and interpretation of intracranial pressure after head injury. Acta Neurochir Suppl 96:114-118

10. Czosnyka M, Czosnyka Z, Momjian S, Pickard JD (2004) Cerebrospinal fluid dynamics. Physiol Meas 25:R51-R76

11. Owler BK, Fong KC, C Z (2001) Importance of ICP monitoring in the investigation of CSF circulation disorders. Br J Neurosurg 15: 439-440

12. Czosnyka M (1996) Testing of cerebrospinal compensatory reserve in shunted and non-shunted patients:a quide to interpretation based on an observationl study. J Neurol Neurosurg Psychiatry 60(5): 549-558

13. Czosnyka M, Pickard JD (2004) Monitoring and interpretation of intracranial pressure. J Neurol Neurosurg Psychiatry 75:813-821

14. Schuhmann MU, Sood S, McAllister JP et al (2008) Value of overnight monitoring of intracranial pressure in hydrocephalic children. Pediatr Neurosurg 44(4):269-279

15. Ekstedt J a N (1978) CSF hydrodynamic studies in man. J Neurol Psychiatry 41:345-353

16. Børgesen SE, Albeck MJ, Gjerris F et al (1992) Computerized infusion test compared to steady pressure constant infusion test in measurement of resistance to CSF outflow. Acta Neurochir (Wien) 119:12-16

17. Nabbanja E, Czosnyka M, Keong NC, Garnett MR, Pickard JD, Lalou AD (2018) Is there a link between ICP-derived infusion test parameters and outcome after shunting in NPH ? Acta Neurochir Suppl 126:229-232

18. Momjian S, Owler BK, Czosnyka Z, Czosnyka M, Pena A, Pickard JD (2004) Pattern of white matter regional cerebral blood flow and autoregulation in normal pressure hydrocephalus. Brain 127(Pt5): 965-972

19. Lalou AD, Czosnyka M, Donnelly J et al (2018) Cerebral autoregulation, cerebrospinal fluid outflow resistance, and outcome following cerebrospinal fluid diversion in normal pressure hydrocephalus. J Neurosurg 38(7):587-592

20. Marmarou A, Maset AL, Ward JD, Chois S, Brooks D, Lutz HA Moulton RJ, Muizelaar JP, DeSalles A, Young H (1987)
Contribution of CSF and vascular factors to elevation of ICP in severely head-injured patients. J Neurosurg 66:883-890

21. Czosnyka M, Piechnik S, Richards HK, Kirkpatrick P, Smielewski P, Pickard JD (1997) Contribution of mathematical modelling to the interpretation of bedside tests of cerebrovascular autoregulation. $\mathrm{J}$ Neurol Neurosurg Psychiatry 63:721-731

22. Donnelly J, Budohoski KP, Smielewski P, Czosnyka M (2016) Regulation of the cerebral circulation : bedside assessment and clinical implications. Crit Care 20(1):129

23. Sykora M, Czosnyka M, Liu X, et al (2016) Autonomic impairment in severe traumatic brain injury: a multimodal neuromonitoring study. 44(6):1173-1181

24. Lemaire JJ, Khalil T, Cervenansky F et al (2002) Slow pressure waves in the cranial enclosure. Acta Neurochir 144:43-254

25. Lalou DA, Czosnyka M, Donnelly J, Lavinio A, Pickard JD, Garnett M, Czosnyka Z (2016) Influence of general anaesthesia on slow waves of intracranial pressure. Neurol Res 38:587-592

26. Czosnyka M, Laniewski W, Batorski L, Zaworski W (1988) Analysis of intracranial pressure waveform during infusion test. Acta Neurochir 93:140-145

27. Lofgren JAN, Zwetnow N (1973) The pressure-volume curve of the cerebrospinal fluid space in dogs. Acta Neurol Scand 49:557-574

28. Swallow DMA, Fellner N, Varsos GV, Czosnyka M, Smielewski P, Pickard JD, Czosnyka Z (2014) Repeatability of cerebrospinal fluid constant rate infusion study. Acta Neurol Scand 130:131-138

29. Tisell M, Edsbagge M, Stephensen H, Czosnyka MWC (2002) Elastance correlates with outcome after endoscopic third ventriculostomy in adults with hydrocephalus caused by primary aqueductal stenosis. Neurosurgery 50:70-77

30. Ekstedt J (1977) CSF hydrodynamic studies in man. 1. Method of constant pressure CSF infusion. J Neurol Neurosurg Psychiatry 40: 105-119

31. Pickard JD, Czosnyka Z, Czosnyka M et al (2009) In: Steiger H-J (ed) Coupling of sagittal sinus pressure and cerebrospinal fluid pressure in idiopathic intracranial hypertension - a preliminary report BT - Acta Neurochirurgica Supplements. Springer Vienna, Vienna, pp 283-285

32. Krishnakumar, Deepa. Pickard JD, Czosnyka Z, Allen L, Parker A (2014) Idiopathic intracranial hypertension in childhood : pitfalls in diagnosis. 56(8):749-55

33. Chari A, Czosnyka M, Richards HK, Pickard JD, Czosnyka ZH (2014) Hydrocephalus shunt technology: 20 years of experience from the Cambridge Shunt Evaluation Laboratory. J Neurosurg 120:697-707

34. Czosnyka Z, Czosnyka M, Owler B et al (2005) Clinical testing of CSF circulation in hydrocephalus. Acta Neurochir Suppl 95:247251

35. Smielewski P, Czosnyka M, Zabolotny W, Kirkpatrick P, Richards H, Pickard JD (1997) A computing system for the clinical and experimental investigation of cerebrovascular reactivity. Int J Clin Monit Comput 14:185-198

36. Kim D, Kasprowicz M, Carrera E (2009) The monitoring of relative changes in compartmental compliances of brain. Physiol Meas 30(7):647-659

37. Kim D-J, Eng M, Czosnyka $Z$ et al (2009) Index of cerebrospinal fluid compensatory reserve in hydrocephalus. Neurosurgery 64 : 494-502

38. Weerakkody RA, Czosnyka M, Schuhmann MU, Schmidt E, Keong N, Santarius T, Pickard JD, Czosnyka Z (2011) Clinical assessment of cerebrospinal fluid dynamics in hydrocephalus. Guide to interpretation based on observational study. Acta Neurol Scand 124:85-98

39. Maksymowicz M, Czosnyka M, Koszewski W, Szymanska A, Traczewski W (1989) The role of cerebrospinal compensatory parameters in the estimation of functioning of implanted shunt system 
in patients with communicating hydrocephalus (preliminary report). Acta Neurochir 101:112-116

40. Eisentrager A, Sobey I, Czosnyka M (2013) Parameter estimations for the cerebrospinal fluid infusion test. Math Med Biol 30:157-174

41. Andersson N (2007) Cerebrospinal fluid infusion methods, online $\mathrm{PhD}$ these at https://www.diva-portal.org/smash/get/diva2:140775/ FULLTEXT01.pdf

42. Andersson N, Malm J, Bäcklund T, Eklund A (2005) Assessment of cerebrospinal fluid outflow conductance using constant-pressure infusion - a method with real time estimation of reliability. Physiol Meas 26:1137-1148

43. Czosnyka M, Smielewski P, Timofeev I, Lavinio A, Guazzo E, Hutchinson P, Pickard J (2007) Intracranial pressure : more than a number. Neurosurg Focus 22:5-11

44. Czosnyka M, Czosnyka Z, Agarwal-harding KJ, Pickard JD (2012) Modeling of CSF dynamics: legacy of Professor Anthony Marmarou. Acta Neurosurg Suppl 113:9-14
45. Robin X, Turck N, Hainard A, Tiberti N, Lisacek F, Sanchez JC, Müller M (2011) pROC: an open-source package for R and S+ to analyze and compare ROC curves. BMC Bioinformatics 12:77

46. Marmarou A, Shulman K (1975) Compartmental analysis of compliance and outflow resistance of the cerebrospinal fluid system. J Neurosurg 43(5):523-534

47. Friedman D (2004) Pseudotumor cerebri. Neurol Clin 22:99-131

48. Stephensen H, Andersson N, Eklund A, Malm J, Tisell M, Wikkelsö C (2005) Objective B-wave analysis in 55 patients with non-communicating and communicating hydrocephalus. J Neurol Neurosurg Psychiatry 76:965-970

Publisher's note Springer Nature remains neutral with regard to jurisdictional claims in published maps and institutional affiliations. 